\title{
Services for Space Mission Support Within The ESA Space Situational Awareness Space Weather Service Network
}

\author{
Donder ED ${ }^{1 *}$, Crosby N ${ }^{1}$, Kruglanski M ${ }^{1}$, Andries $\mathrm{J}^{2}$, Devos A $^{2}$, Perry $\mathrm{C}^{3}$, Borries $\mathrm{C}^{4}$, Martini $\mathrm{D}^{5}$, Glover $\mathrm{A}^{6}$ and Luntama JP
}

${ }^{1}$ Royal Belgian Institute for Space Aeronomy, Uccle, Belgium

${ }^{2}$ Royal Observatory of Belgium, Uccle, Belgium

${ }^{3}$ STFC RAL Space, UK

${ }^{4}$ Institute of Communications and Navigation, German Aerospace Centre, Neustrelitz, Germany

${ }^{5}$ Norwegian Center for Space Weather/Tromsø Geophysical Observatory, Norway

${ }^{6}$ ESA SSA Programme Office European Space Operation Centre Darmstadt, Germany

\begin{abstract}
Spacecraft operations are by nature complex and every satellite's operational environment poses a range of potential risks, often a unique combination for a given orbit. The implications of interruptions of operations, data transfer and service provision, are serious, both in terms of cost and capability, thus it is imperative to mitigate against all operational risks to the fullest extent possible.

In the frame of its Space Situational Awareness (SSA) programme, the European Space Agency (ESA) is establishing a Space Weather Service Network to support end-users, in a wide range of affected sectors, in mitigating the effects of space weather on their systems, reducing costs and improving reliability. This service network is currently in a test and validation phase and encourages user engagement and feedback.

The network is organised around five Expert Service Centres (ESCs) focusing on Solar Weather, Heliospheric Weather, Space Radiation Environment, lonospheric Weather and Geomagnetic Conditions. Each ESC is connecting different expert groups, federating their space weather products, and ensuring the quality and consistency of the provided information. The service network also includes a central Data Centre and the SSA Space Weather Coordination Centre (SSCC).
\end{abstract}

In this presentation we give an overview of the current status of the network (http://swe.ssa.esa.int/), the targeted end-user groups and Expert Service Centres with a focus on the space community.

Keywords: Space weather; Space situational awareness; Service network

\section{Introduction}

The objective of the ESA Space Situational Awareness (SSA) programme is to support Europe's independent utilisation of, and access to, space through the provision of timely and accurate information, data and services regarding the space environment, and particularly regarding hazards to infrastructure in orbit and on the ground.

The SSA programme will, ultimately, enable Europe to autonomously detect, predict and assess the risk to life and property due to man-made space objects (remnant debris, re-entries, in-orbit explosions and release events, in-orbit collisions, disruption of missions and satellite-based service capabilities), potential impacts of NearEarth Objects (NEOs), and the effects of space weather phenomena on space- and ground-based infrastructure.

The programme is currently in its Period Two [1] and is active in three main areas:

- Survey and tracking of objects in Earth orbit.

- Monitoring and forecasting space weather.

- Watching for NEOs

Within this framework, the focus in the Space Weather Segment (SWE) of the SSA Programme is on developing a system capable of providing space weather services to end users. The system under development is based on a federated architecture where the service provision will be carried out by Expert Service Centres (ESCs) in the
Programme Member States. These collaborative centres bring together European expertise and assets. Five ESCs focusing on Solar Weather, Heliospheric Weather, Space Radiation Environment, Ionospheric Weather and Geomagnetic Conditions are being established. Space weather services from the ESA SSA system are being made available to the end users because of a framework set to incorporate service level agreements, leading to both a substantial extension of products available and improved reliability of provision. In parallel to the provision of the current services, the ESCs are introducing new, innovative services and developing the necessary processes in order to provide these in a reliable manner. Consequently, the SWE network of services is expected to grow substantially within SSA Period 2, particularly within the context of the ongoing Expert Service Centre Definition and Development (P2-SWE-I) activity taking place during 2015-2017. The overall structure of the network is shown in Figure 1.

For the development of the SWE service network, two distinct

*Corresponding author: Donder ED, Royal Belgian Institute for Space Aeronomy, Uccle, Belgium, Tel: +32 237304 04; E-mail: erwin.dedonder@aeronomie.be

Received September 01, 2016; Accepted January 02, 2017; Published January 05, 2017

Citation: Donder ED, Crosby N, Kruglanski M, Andries J, Devos A, et al. (2017) Services for Space Mission Support Within The ESA Space Situational Awareness Space Weather Service Network. J Aeronaut Aerospace Eng 6: 180. doi: 10.4172/2168-9792.1000180

Copyright: @ 2017 Donder ED, et al. This is an open-access article distributed under the terms of the Creative Commons Attribution License, which permits unrestricted use, distribution, and reproduction in any medium, provided the original author and source are credited. 


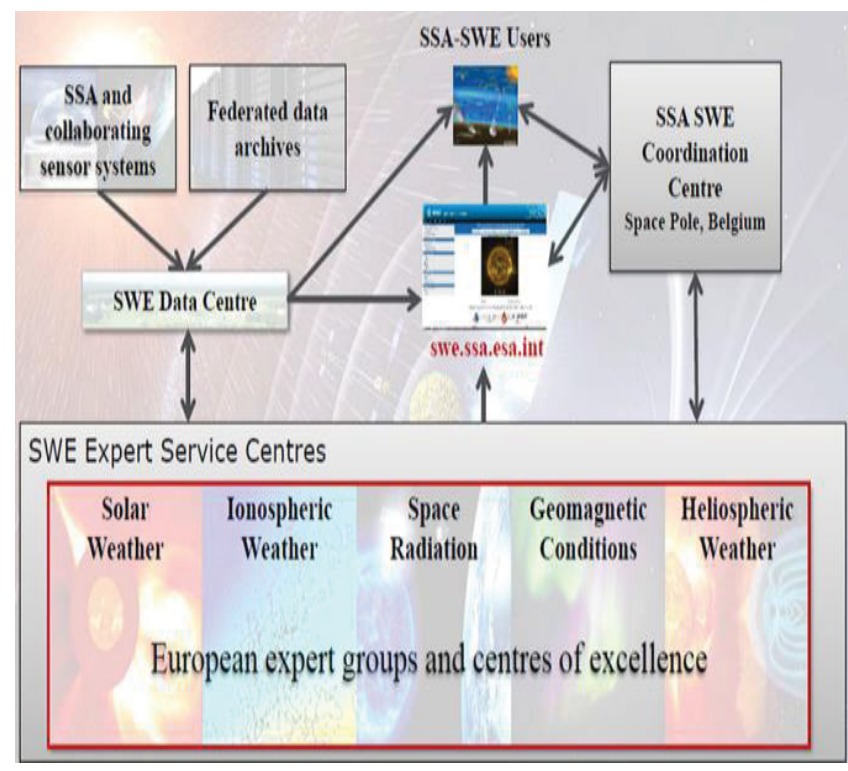

Figure 1: SSA-SWE service network structure [1].

approaches are followed. The first approach is a bottom-up approach where existing space weather assets in Europe are deployed and further developed into a set of representative and essential precursor services. The deployment and operation of this set of precursor services allows to gather feedback from the user community, to make early assessments of the network and to provide key inputs for its strategic service development roadmaps. The second approach is a top-down approach starting with the definition of the customer team also organises visits to the SSCC premises upon invitation and/or request (helpdesk.swe@ ssa.esa.int).

In what follows, for each ESC their mission statement, contributing expert groups, provided services and corresponding user types and provided products are described. The SSCC tailoring of some products for space mission support is also described (Figure 1).

Requirements [2] from which system requirements [3] have been derived in succession. The top down design process benefits from the experience and feedback gained from the pre-operation of the SWE service network set up by the first approach. The second approach has defined eight service domains - Spacecraft Design (SCD), Spacecraft Operation (SCO), Human Space Flight (SCH), Launch Operation (LAU), Transionospheric radio link (TIO), SSA Space Surveillance and Tracking (SST), Non-space systems operation (NSO), and General data service (GEN) - for which 39 services have been identified (Refer Appendix).

Since space weather is a rapidly evolving domain, with users gaining more in-depth knowledge of how space weather affects their systems and new user groups becoming aware of the potential impact of space weather, it is important to establish a close relationship with the different potential user communities. Such a relationship enables user awareness and ensures its progress from awareness, to agreement, and from agreement to adoption of evidence-based practices. In the approach to establish a close relationship with the end-users of space weather services, the SSA Space Weather Coordination Centre (SSCC) - which provides the user helpdesk and first line user support for the SWE network conducts three main actions:

- Bringing the network to the users to increase its awareness,
- Identifying key persons within the different user communities

- Setting up end user meetings to learn the user requirements and to translate them into a user tailored space weather service.

Members of the SSCC team attend workshops, conferences etc. to promote the network and to meet end users, and the team also organises visits to the SSCC premises upon invitation and/or request (helpdesk.swe@ssa.esa.int).

In what follows, for each ESC their mission statement, contributing expert groups, provided services and corresponding user types and provided products are described. The SSCC tailoring of some products for space mission support is also described.

\section{The Expert Service Centre Solar Weather}

\section{Mission statement}

The mission of the Solar Weather Expert Service Centre (S-ESC) is to provide and develop the functionalities, capabilities and expertise in the domain of Solar Weather that are needed within the ESA SSA SWE Network to achieve as a collaborative enterprise its mission of demonstrating and assessing the influences of Space Weather and informing and supporting end-users through the provision of accurate, reliable and timely products and (pre) operational services, tailored to their requirements [4-15].

The S-ESC thus provides, implements and supports the Solar Weather products and capabilities of the ESA SSA SWE. This includes the observation, monitoring, interpretation, modelling and forecasting of Solar Weather conditions with an emphasis on Solar (sub)-surface and Solar coronal features, events and processes that drive Space Weather in our Solar System.

\section{Expert groups}

The current network of Expert Groups contributing to the S-ESC network is:

- Solar Influence Data analysis Centre (SIDC), Royal Observatory of Belgium (ROB), Belgium.

- Kanzelhöhe Observatory (KSO), University of Graz (UniGraz), Austria.

- Research Center for Astronomy and Applied Mathematics, Academy of Athens, Greece.

- Osservatorio Astrofisico di Catania (CAO), Istituto Nazionale di Astrofisica (INAF), Italy.

- Institute of 4D Technologies, University of Applied Sciences North Western Switzerland.

\section{Services and user types}

The functionalities and responsibilities of the S-ESC are located at the start of the causality chain of Space Weather events starting from the Solar origin up to the effects on Geospace, Earth and, eventually, the impact on user infrastructure. Hence, the focus of the S-ESC is very much on the production and assessment of synoptic Solar data.

Solar Weather products can have direct relevance to the end-user (e.g. a general probabilistic forecast on the likelihood of solar events given the current Solar Weather conditions). In addition, Solar Weather ESC products are often digested by other Centres that, based on their subsequent expertise in the physical systems impacted, further specify and predict the more precise characteristics of these impacts [16-20]. 
Citation: Donder ED, Crosby N, Kruglanski M, Andries J, Devos A, et al. (2017) Services for Space Mission Support Within The ESA Space Situational Awareness Space Weather Service Network. J Aeronaut Aerospace Eng 6: 180. doi: 10.4172/2168-9792.1000180

\section{Products}

Below we give a list of products provided by the S-ESC, illustrated with the CACTus CME detection (Figure 2) and a full disk magnetogram with eligible active regions (Figure 3) produced by Athens Effective Solar Flare Forecasting (AEFFort).

\section{a. Solar imaging}

- White light continuum images from ground based stations (SIDC/USET, UniGraz/KSO, INAF/CAO).

- H-Alpha images from ground based stations (USET, KSO, CAO).

- PROBA2/SWAP EUV images.

- European mirror of SDO imagery.

b. Spectral radio observations

- Radio spectra from the Humain radio station, Belgium.

- Radio spectra from the eCallisto Global network.

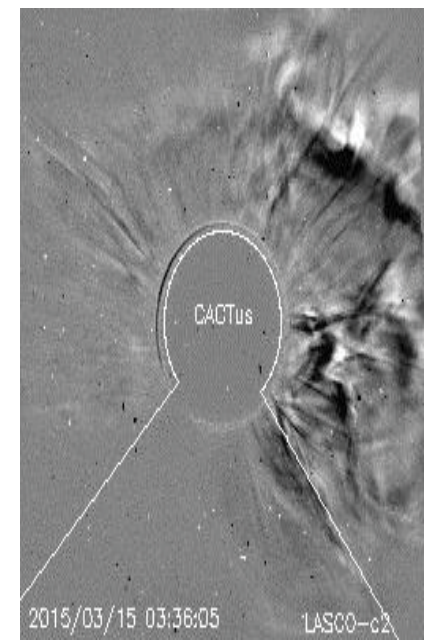

Figure 2: Cactus CME detection in SOHO/LASCO coronagraphic image [4,5].

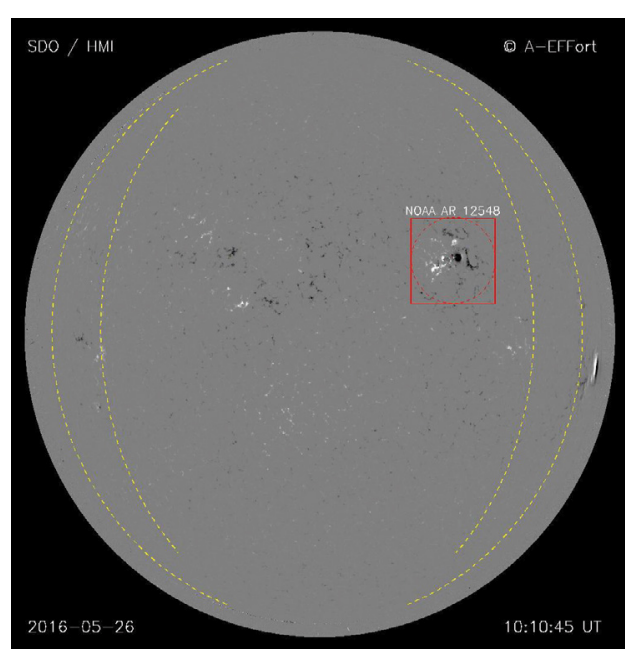

Figure 3: Eligible active regions identified on full-disk SDO/HMI line-of-sight magnetogram (from A-Effort) [6-8].

\section{c. Solar irradiance}

- PROBA2/LYRA 4channel (E) UV irradiance.

- Recalibrated LYRA data as proxy for X-ray flare magnitudes.

\section{d. Solar feature and event detections/characterisation}

Characterisation of Sunspot Groups: from USET and CAO.

Automated Radio Burst detections on SIDC/Humain radio observations.

- (CACTus) Automated CME detection in SoHO/LASCO coronagraphic images.

- Optical flare detections from KSO.

-X-ray flare detections based on NOAA/GOES flux measurements.

- Filament detections from KSO.

- Human operator $(7 \mathrm{~d} / 7 \mathrm{~d}, 16 \mathrm{~h} / 24 \mathrm{~h})$ moderation and expert annotation and alerting complementing the above automated event detections.

- Solar Activity indices.

- International Sunspot Number.

- Solar activity and indices forecasts.

-3-day forecast of radio flux F10.7 index.

- Probabilistic Solar Flare predictions (2 distinct methods).

\section{e. Service products}

- Email alerts associated to the feature and event detections.

- Image browsing with Solar Active Region annotations.

- All quiet Alert: marking times with exceptionally low Space Weather risk. This product is targeted at Satellite operators.

- Daily bulletin on Solar (and Heliospheric and Geomagnetic) Weather (Human operator generated).

\section{The Expert Service Centre - Heliospheric Weather}

\section{Mission statement}

The mission of the Heliospheric Weather Expert Service Centre (H-ESC) is to provide and develop the functionalities, capabilities and expertise in the domain of Heliospheric Weather that are needed within the ESA SSA SWE network to achieve as a collaborative enterprise its mission of demonstrating and assessing the influences of Space Weather and informing and supporting end users through the provision of accurate, reliable and timely products and (pre-) operational services, tailored to their requirements.

The specific goal of the H-ESC is to fully exploit heliophysics assets and expertise from Europe, and further afield, to provide, improve and support the provision of alerts, forecasts and post event analysis of space weather conditions both near to the Earth and at other locations within the heliosphere.

This will be accomplished through the use of remote-sensing and in situ monitoring to identify and track key transient features (including coronal mass ejections, high-speed solar wind streams and solar energetic particles), combined with the use of advanced modelling and analysis techniques to predict their arrival at various points of interest in the heliosphere. 
Citation: Donder ED, Crosby N, Kruglanski M, Andries J, Devos A, et al. (2017) Services for Space Mission Support Within The ESA Space Situational Awareness Space Weather Service Network. J Aeronaut Aerospace Eng 6: 180. doi: 10.4172/2168-9792.1000180

Page 4 of 11

\section{Expert groups}

The H-ESC is being newly established as part of the ESA SSA SWE Period 2 ESC definition and development activities that kicked-off towards the end of September 2015. The initial H-ESC network consists of eight Expert Groups of which five will be providing products and the remaining three are consultants with specialised knowledge in the areas of CME characterisation, service assessment and development and modelling which are key areas for the initial development. The H-ESC network coordination is provided by STFC RAL Space, UK. The full list of H-ESC Expert Groups is:

- STFC RAL Space, UK (Coordination, scientific QA and service assessment).

- UK Met Office, UK (operational forecasting service, MHD modelling).

- University of Graz, Austria (solar wind and CME propagation).

- DTU, Denmark (near-Earth solar wind transient detection).

- IRAP, France (AMDA and propagation validation tools).

- KU Leuven, Belgium (European modelling assessment).

- DH Consultancy, Belgium (Existing service assessment).

- University of Göttingen, Germany (CME propagation).

\section{Services and user types}

The H-ESC has responsibility for three service areas falling within the general data, spacecraft operations and spacecraft design service domains:

- GEN/mod - End-to-end modelling capability for space weather.

- SCO/pla - Spacecraft operations for interplanetary missions.

- SCD/pla - Spacecraft design for interplanetary missions.

The SCO/pla and SCD/pla are two new service areas that are being defined as part of the current P2-SWE-I activity with a specific focus on the support of ESA interplanetary science missions.

\section{Products}

With the initial development of the H-ESC the focus is on the provision of products that address the heliospheric forecasting, nowcasting and alerting needs of the users and other ESCs with respect to background solar wind and solar wind transients and their arrival in the vicinity of the Earth. During the second part of the current project the products will be extended to provide broader support for heliospheric weather for other locations within the heliosphere.

The initial set of products to be released during the first half of 2016 consists of:

- Forecasts of near-Earth Solar wind based on MHD (WSA/ENLIL) and empirical modelling techniques and with lead times of 4 to 7 days depending on the model.

- Nowcasts of the near-Earth Solar wind measurements to provide information on current conditions and for verification and assessment of forecasts.

- Predictions of near-Earth CME arrival times based on MHD and empirical modelling techniques of the propagation from the Sun to the Earth.
- Near-Earth energetic particle measurements from GOES for SEP arrival assessment.

- Near-Earth space weather alerts based on forecaster and automated assessment of the near-Earth heliospheric weather conditions including shock, ICME and SIR arrival.

The second set of products that will be released during the autumn of 2016 are:

- Forecast of Solar wind at other locations in the heliosphere based on MHD modelling techniques.

- Tailored CME arrival predications for other locations within the heliosphere based on MHD and drag based modelling techniques.

- Tailored heliospheric weather alerts for other locations within the heliosphere.

Figure 4 shows an example of the WSA/ENLIL MHD heliospheric modelling. Empirical modelling products are shown in Figure 5 (left: coronal hole based near-Earth solar wind estimates, right: drag based model forecast of CME arrival.

In addition the H-ESC will provide a range of ancillary and support products and capabilities including:

- Case study and special event modelling.

- Assessment report on near-Earth forecast and alerting performance.

- Archive of H-ESC products.

- Integrated access to science archive (CDPP, UKSSDC) on nearEarth and interplanetary measurements in support of post event analysis.

- Long term statistical products on heliospheric weather conditions.

\section{The Expert Service Centre - Space Radiation}

\section{Mission statement}

The mission of the Space Radiation ESC (R-ESC) is to provide and develop the functionalities, capabilities and expertise in the domain of Space Radiation that are needed within the ESA SSA SWE Network to achieve as a collaborative enterprise its mission of demonstrating and assessing the influences of Space Weather and informing and

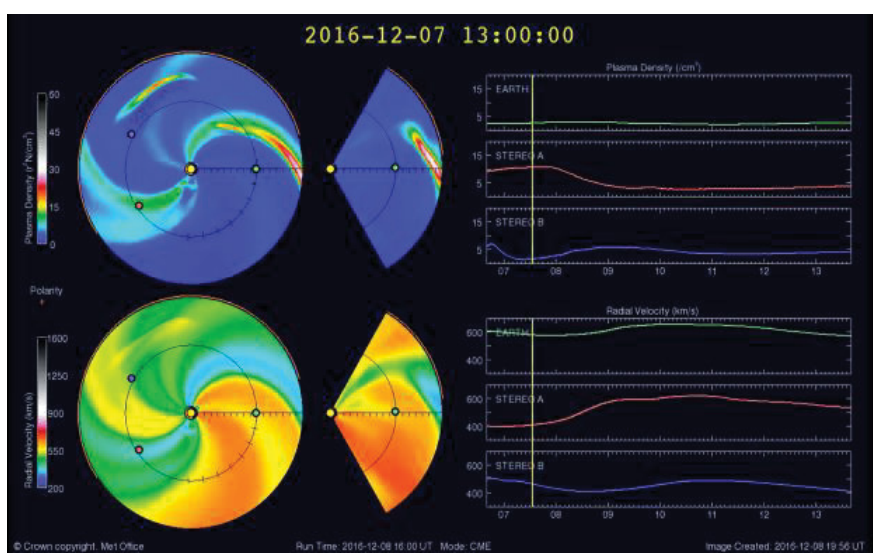

Figure 4: Example of the WSA/ENLIL MHD heliospheric modelling that is being provided by UK Met Office as part of the H-ESC forecasting centre [9]. 

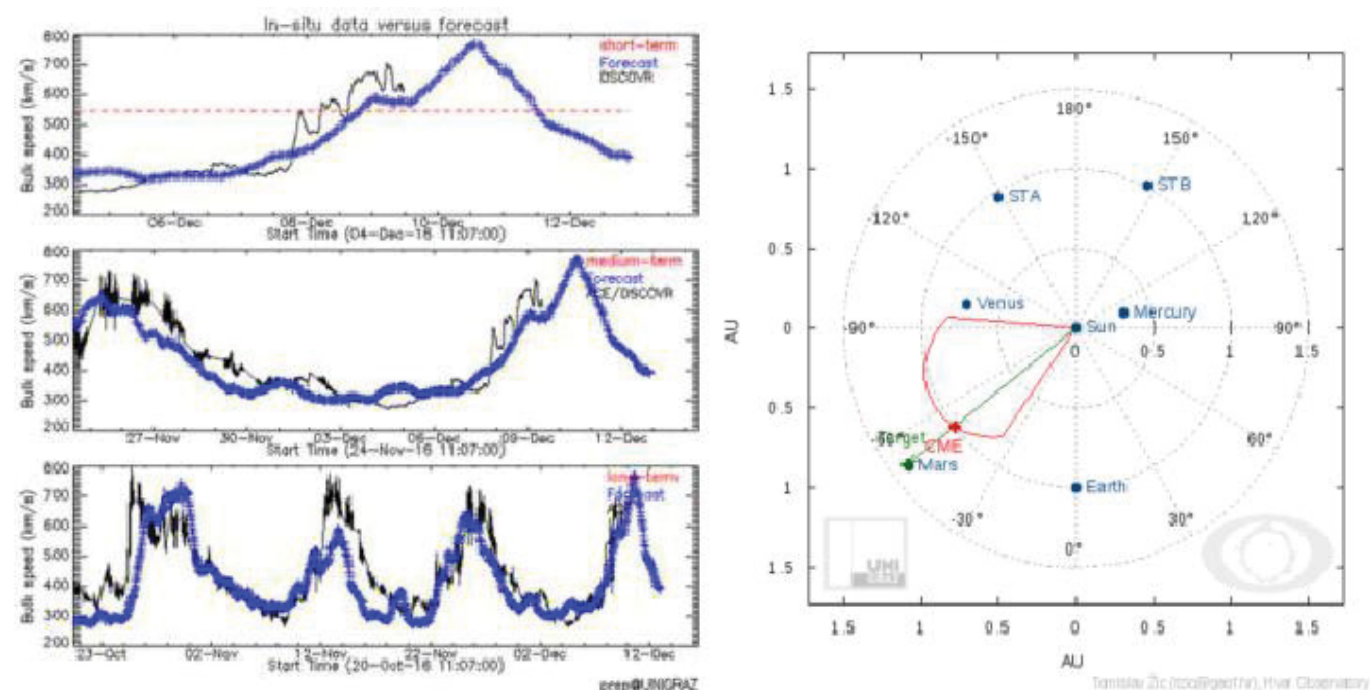

Figure 5: Example of the empirical modelling products provided by University of Graz. (left) coronal hole based near-Earth solar wind estimates [10], (right) drag based model forecast of CME arrival [11].

supporting end users through the provision of accurate, reliable and timely products and (pre-) operational services, tailored to their requirements.

The R-ESC ensures that the monitoring, modelling and forecasting of space particle radiation (ambient plasma, solar energetic particles, radiation belts, galactic cosmic rays), micron-size particulates (from meteoroids and space debris), as well as all types of phenomena induced effects on technologies and biological systems, are fully covered in regard to the near-Earth space environment.

\section{Expert groups}

The R-ESC is currently a network of eleven Expert Groups comprised of eight product providers and three consultants with expertise in charged particle radiation. Expert group names and their associated countries in alphabetical order are listed below. During P2SWE-I the R-ESC is being coordinated by the Royal Belgian Institute for Space Aeronomy (BIRA-IASB).

\section{a. Providers}

- Radiation Hardness Assurance and Space Weather (RAS), Seibersdorf Laboratories Gmbh (Austria).

- Paul Buehler (Austria).

- Centre for Space Radiations (CSR), Université Catholique de Louvain (Belgium).

- Space Physics Division (BIRA-IASB), Royal Belgian Institute for Space Aeronomy (Belgium).

-Space Research Laboratory (SRL), University of Turku (Finland).

-Department Radiation Biology (DLR-IAM), DLR Institute of Aerospace Medicine (Germany).

-Athens Neutron Monitor Station (ANeMoS), National and Kapodistrian University of Athens (Greece).

-Mullard Space Science Laboratory (MSSL), University College London (United Kingdom).

\section{b. Consultants}

- Ondrej Santolik, Institute of Atmospheric Physics (IAP) (Czech Republic).

- Ilya Usoskin, Sodankylä Geophysical Observatory (SGO), University of Oulu (Finland).

- Clive Dyer, CSDRadConsultancy Ltd (United Kingdom).

\section{Services and user types}

Seventeen services from five of the service domains introduced in section I fall under the responsibility of the R-ESC. Domains and their respective services are listed here:

\section{Spacecraft design}

- SCD/arv - Data archive.

- SCD/orb - In orbit verification.

- SCD/pst - Post event analysis.

\section{Spacecraft operation}

- SCO/orb - In orbit environment and effects monitoring.

- SCO/pst - Post event analysis.

- SCO/for - In-orbit environment and effects forecast.

- SCO/ana - In-orbit mission risk analysis.

\section{Human space flight}

- $\mathrm{SCH} /$ orb - In-flight crew radiation exposure.

- $\mathrm{SCH} / \mathrm{pstb}$ - Cumulative crew radiation exposure.

- SCH/for - Increased crew radiation exposure risk.

\section{Launch operation}

- LAU/orb - In-flight monitoring of radiation effects in sensitive electronics.

- LAU/pst - Estimate of radiation effects in sensitive electronics. 
Citation: Donder ED, Crosby N, Kruglanski M, Andries J, Devos A, et al. (2017) Services for Space Mission Support Within The ESA Space Situational Awareness Space Weather Service Network. J Aeronaut Aerospace Eng 6: 180. doi: 10.4172/2168-9792.1000180

Page 6 of 11

- LAU/for - Forecast of radiation storms.

- LAU/drg - Atmospheric density forecast.

- LAU/ios - Risk estimate of service disruption caused by ionospheric scintillations.

- LAU/mcp - Risk estimate of microparticle impacts.

\section{Non-space systems operations}

\section{- NSO/air - Service to airlines}

New products being provided during P2-SWE-I (see Section IV.D) were specifically chosen to improve these four services: SCD/ arv, SCD/pst, SCO/orb, and NSO/air. During P2-SWE-I it is also being determined to what extent the seventeen R-ESC services as specified in the SSA SWE Customer Requirements document can be fully provided on the basis of existing European capabilities. This is done by reviewing existing capabilities (assets), analysing where there are gaps and when necessary identifying development needs. The Space Radiation ESC network expansion is thus directly linked to the latter.

\section{d. Products}

During P2-SWE-I the R-ESC continues to provide users with seven existing products that were delivered during the SSA Preparatory Programme. These products are available on the R-ESC homepage on the SSA SWE portal (http://swe.ssa.esa.int/web/) and are listed below:

- Radiation exposure estimation at aircraft altitude (AVIDOS).

- GLE alert service.

- Multi-station Neutron Monitor data.

- Space Environment Information System (SPENVIS).

- Space Environment Data System (SEDAT).

- Environment Information System for Operations (SEISOP).

- European Debris Impact Database (EDID).

Figure 6 displays two of these products, respectively the GLE alert service (upper panel) and AVIDOS that provides radiation exposure estimation at aircraft altitudes (lower panel).

During P2-SWE-I 28 new products will be delivered, implemented and provided to users, also via the SSA SWE portal (http://swe.ssa.esa. int/web/). These products can broadly be classified as follows:

- PROBA-V / EPT e, p, He flux (spectra time series, geographical maps).

- PROBA-V / EPT energy spectra (auroral electron, SAA p and He).

- SREM data on Proba-1, Integral, Rosetta, Herschel, Planck.

- Radiation and accumulated doses at ISS.

- SEP post-event analysis for aviation radiation exposure.

- Very high-energy SEP environment (proton fluence, worst-case proton flux).

- SEP event catalogue.

- Electron radiation belt models (GEO, MEO, LEO).

- Coronal Mass Ejections and Solar Energetic Particles (COMESEP) Alert System.
- Solar Energetic Particle Environment Modelling (SEPEM) Application Server.

- Plasmasphere electron density distribution model.

Existing as well as new products provide users a large spectrum of applications ranging from technologies to biological systems covering different orbits as well as airline altitudes (Figure 7).

\section{The Expert Service Centre - Ionospheric Weather}

\section{Mission statement}

The mission of the Expert Service Centre Ionospheric Weather (I-ESC) is to provide and develop the functionalities, capabilities and expertise in the domain of ionosphere and upper atmosphere weather that are needed within the ESA SSA SWE Network to achieve as a collaborative enterprise its mission of demonstrating and assessing the influences of Space Weather and informing and supporting end-users through the provision of accurate, reliable and timely products and (pre-) operational services, tailored to their requirements.

\section{Expert groups}

Currently, nine expert groups are contributing by product provision or development to the services for GNSS users in the I-ESC. An overview of these expert groups is shown in Figure 8.

The coordination of the I-ESC is performed by DLR. The currently nine expert groups are complemented during Period 2 through new expert groups, data and products. An I-ESC advisory board is constantly supporting the I-ESC coordinator in his duty to organize the development and operation of the I-ESC services. The vision of the ESC includes a continuous network extension and development. Therefore, the initial assets and expert groups involved in the ESC

\section{Welcome to the European Neutron Monitor Service} for further products and information please vist the options at the right

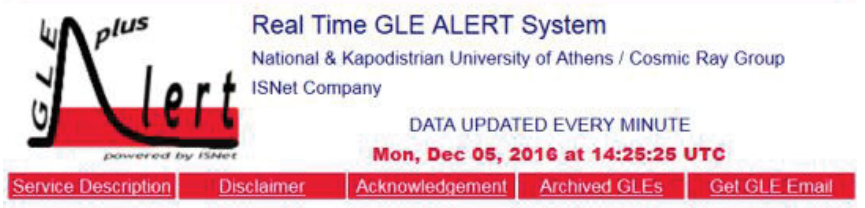

\begin{tabular}{|c|c|c|}
\hline General Alert Status & \multicolumn{2}{|c|}{ Stations Summary } \\
\hline \multirow{4}{*}{ QUIET } & EABa: $[00]$ & Total [ 34 ] \\
\hline & WARNING $[00]$ & Real Time [20] \\
\hline & EWATCH $[00]$ & Near Real Time [ 05 ] \\
\hline & QUIET [34] & Not in Real Time [ 09 ] \\
\hline
\end{tabular}

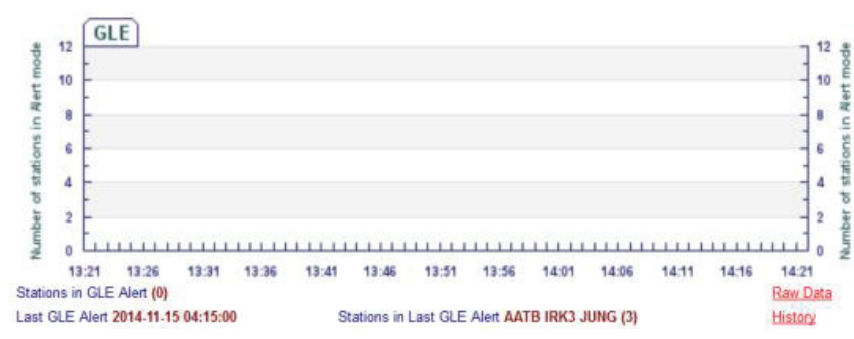

Figure 6: Example of two space radiation ESC products. Upper panel: GLE alert service provided by the University of Athens [12]. Lower panel: Radiation exposure estimation at aircraft altitude (AVIDOS) provided by Seibersdorf Laboratories [13]. Courtesy of the ESA SWE Portal (http://swe.ssa.esa.int/ space radiation). 

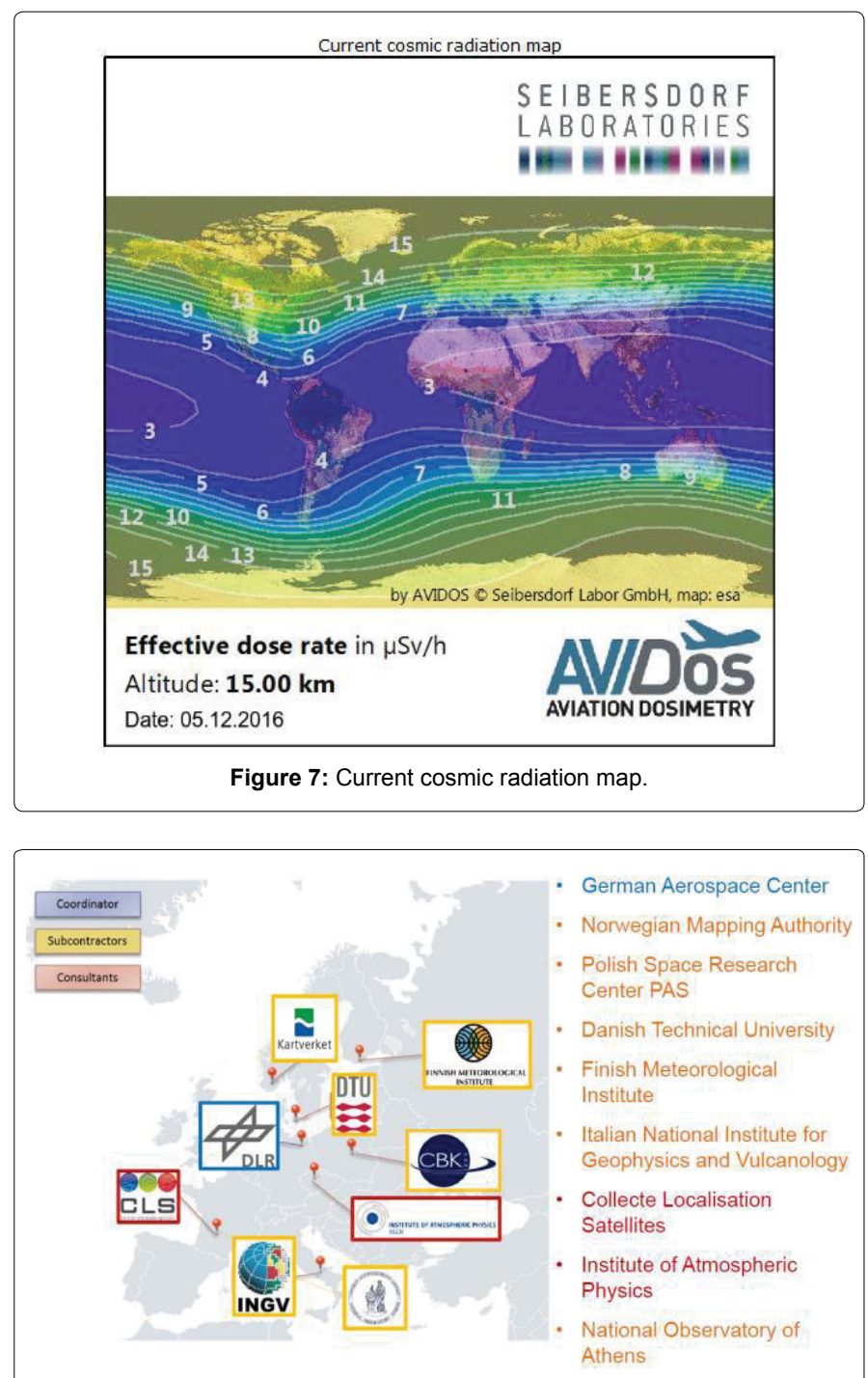

Figure 8: Overview of currently contributing expert groups in the I-ESC.

will be complemented by additional experts and assets extending the network and integrating additional and new products into the I-ESC.

\section{Services and user types}

The I-ESC is responsible for a number of services in the Transionospheric Radiolink (TIO) domain and the SWE services to the Space Surveillance and Tracking (SST) domain. In the TIO domain the I-ESC is providing services mainly to different kinds of GNSS users:

- Users of GNSS single frequency services with average accuracy, no integrity (e.g. typical GNSS mass market user).

- Users of GNSS single frequency services with average accuracy, using integrity (e.g. EGNOS user).

- Users of multi-frequency GNSS systems with average multifrequency accuracy, no integrity (commercial services, public regulated services).

- Users of multi-frequency GNSS systems with average accuracy, integrity (aeronautical multi-frequency).
- Users or multi-frequency GNSS systems with very high accuracy (e.g. GNSS geodetic users, Real-Time Kinematics).

- Users of satellite data communications with high availability / continuity (e.g. Search-and-Rescue, Air Traffic Control/Management via Satellite, high availability/continuity data networks such as Galileo Ground Segment Data Network).

- Other space-based services/products users affected by the ionosphere (UHF - C-band radars, GNSS-R altimetry, UHF/low microwave radio astronomy and deep space communications). For these users, the I-ESC is providing the following services:

- TIO/tcr - Provide near real-time Total Electron Content (TEC) maps.

- TIO/tcf - Provide forecast TEC maps.

- TIO/qua - Provide information on whether standard corrections to GNSS signal are applicable (Quality assessment of ionospheric correction).

- TIO/sci - Provide near real-time estimate of ionospheric scintillation maps.

- TIO/for - Provide estimate of the occurrence risk of ionospheric disturbances (monitoring and forecast of ionospheric disturbances).

The SWE services targeting the the SST domain are mainly addressed to users in the following areas:

- Surveillance and tracking centre(s), stations and services.

- Spacecraft operators.

- Collision warning services.

- Re-entry risk assessment services.

For these users the I-ESC will provide the following services:

- SST/atm - Provide estimates of atmospheric density in the past years and predicted in near real-time.

- SST/arv - Provide a database of past values of solar and geomagnetic indices relevant to drag calculation.

- SST/for - Provide forecast of geomagnetic and solar induces for drag calculation

- SST/ion - Provide now cast of ionospheric group delay to estimate effects on radar signals.

\section{Products}

The I-ESC provides a large variety of products serving the needs of the addressed users and contributing to the provision of the mentioned services. The relation between the products and services is described in the documents [2] and [3] and will be presented in this way on the I-ESC homepage on the SSA SWE portal (http://swe.ssa.esa.int/ web/). Here, currently diverse products in the following categories are provided:

- TEC maps.

- Scintillation maps.

- URSI parameters (ionosonde measurements).

- Databases of solar and geomagnetic indices.

- Ionospheric disturbances. 
Citation: Donder ED, Crosby N, Kruglanski M, Andries J, Devos A, et al. (2017) Services for Space Mission Support Within The ESA Space Situational Awareness Space Weather Service Network. J Aeronaut Aerospace Eng 6: 180. doi: 10.4172/2168-9792.1000180

- Event based alarms for ionospheric disturbances.

A small subset of I-ESC products is shown in Figure 9.

\section{The Expert Service Centre - Geomagnetic Conditions} Mission statement

The mission of the Geomagnetic Conditions Expert Service Centre (G-ESC) is to provide and develop the functionalities, capabilities and expertise in the domain of geomagnetism that are needed within the ESA SSA SWE Network to achieve as a collaborative enterprise its mission of demonstrating and assessing the influences of Space Weather and informing and supporting end-users through the provision of accurate, reliable and timely products and (pre-) operational services, tailored to their requirements.

The G-ESC thus provides implements and supports the geomagnetic products and capabilities of the ESA SSA SWE network for monitoring, interpreting, and forecasting variations of the geomagnetic field on various timescales, relying on in situ ground-based, as well as spaceborne measurements and related derived quantities and models.

\section{Expert groups}

Currently the G-ESC comprises the following eight expert groups:

- Technical University of Denmark - DTU Space.

- Finnish Meteorological Institute (FMI).

- Swedish Institute for Space Physics (IRF).

- German Research Centre for Geosciences (GFZ).

- Polar Geophysical Institute (PGI).

- Solar Influences Data Analysis Center (SIDC).

- Norwegian Mapping Authority (NMA).

\section{Services and user types}

Four user domains have been identified that fall under the responsibility of the G-ESC within the Non-Space System Operators Services (NSO) domain:

- NSO/pow - Service to power system operators (high priority).

- NSO/ppl - Service to pipeline operators (low priority).

- NSO/res - Service to resource exploitation system operators (high priority).

- NSO/tou - Service to auroral tourism sector (medium priority).

\section{Products}

The list of existing and new (that will be delivered during P2SWE-I) products provided by the G-ESC are displayed in Table 1. In Figures 10 and 11 respectively, a 27 days forecast of the daily global Ap index and 30-minutes $\mathrm{dB} / \mathrm{dt}$ forecast map are displayed.

\section{Tailoring of Space Weather Products}

To address specific requests from key users, SSCC is tailoring products of the SWE network into dedicated space weather bulletins. In particular such bulletins have been generated in support to the following ESA missions:

- Launch of GAIA spacecraft (16-20 December 2013) and L2 insertion manoeuvre (6-14 January 2014).

- Last aerobraking campaign of Venus Express (May-July 2014).

- Rosetta mission (since October 2014) including the landing of the Rosetta's sounder Philae on comet 67P/Churyumov-Gerasimenko (12 November 2014).

- Launch of the experimental spaceplane IXV (11 February 2015).

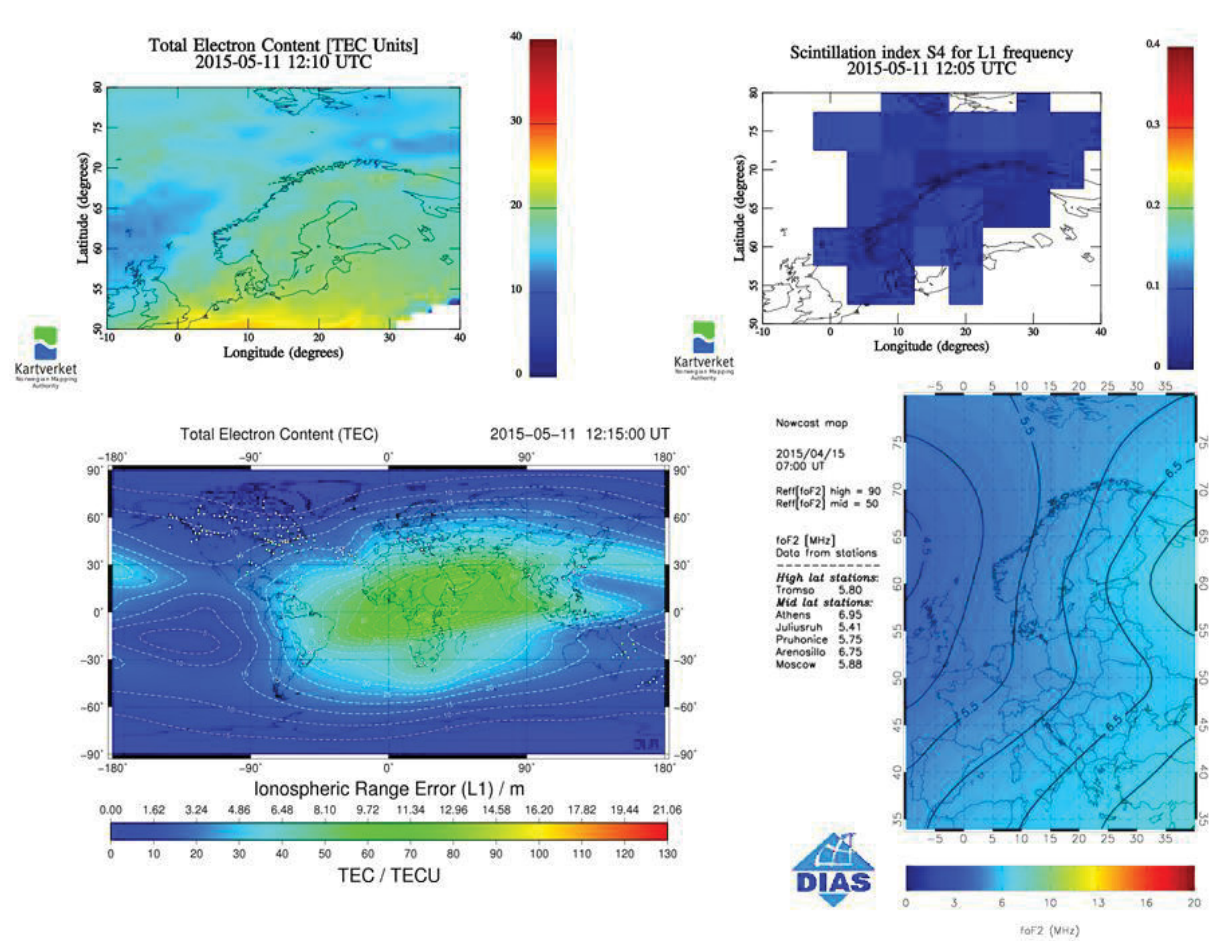

Figure 9: Sample subset for ionospheric products provided in the I-ESC. Upper left: DLR TEC map [14], Upper right: NMA S4 map [15], lower left: NMA TEC map [15], lower right: NOA Now-cast foF2 map [16-18]. 


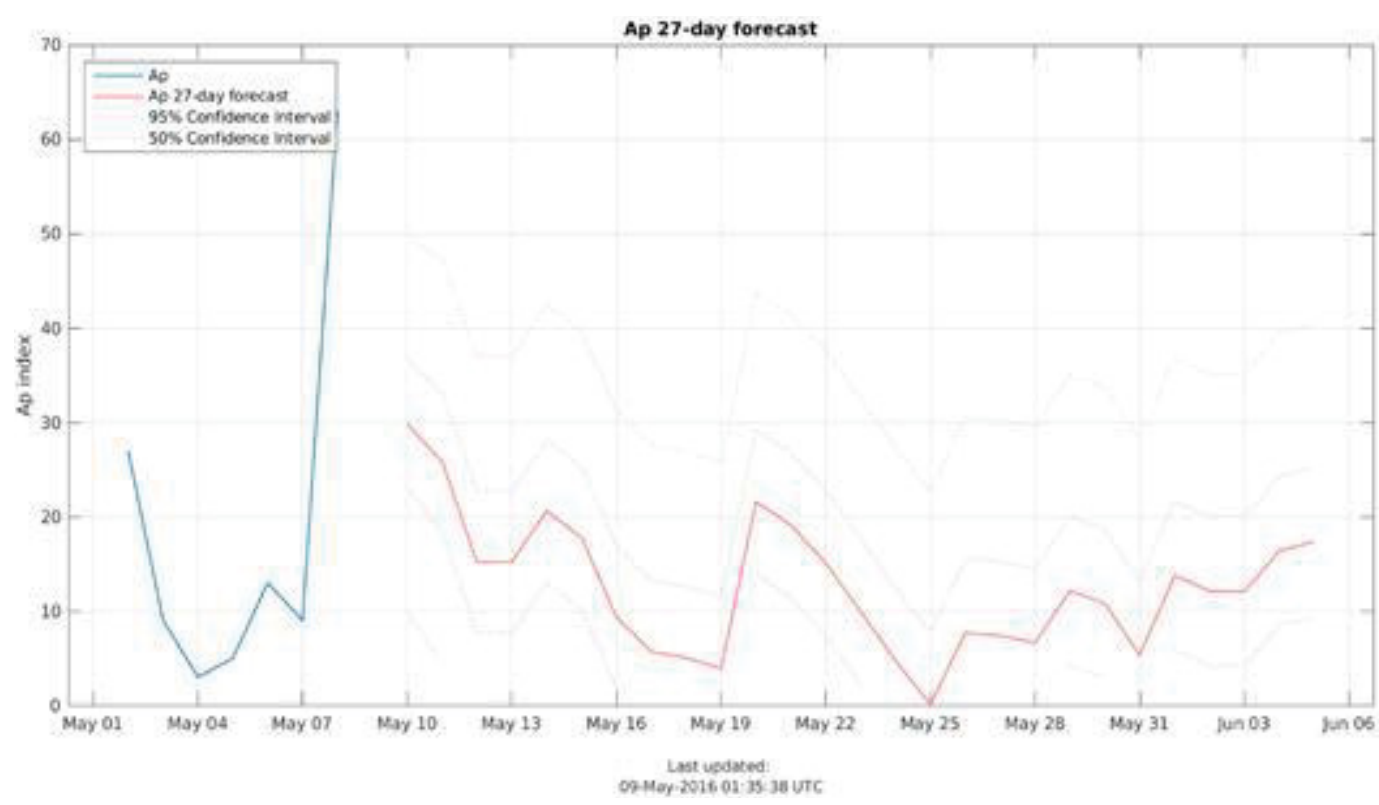

Figure 10: The 27 days forecast of the daily global Ap index, provided by the Norwegian Centre for Space Weather/Troms $\varnothing$ Geophysical Observatory at UiT [19]. In the figure the solid blue line depicts the Ap index data. The forecast, using linear prediction filter, is shown as solid red line with the corresponding upper and lower $(50 \%$ and $95 \%)$ confidence limits in dotted lines.

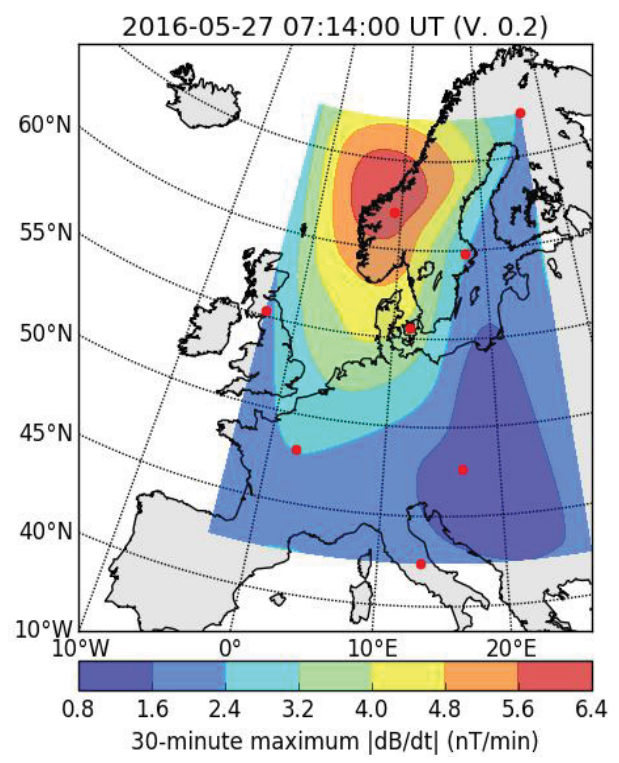

Figure 11: 30-minutes dB/dt forecast map, provided by the Swedish Institute of Space Physics (IRF-Lund) [20]. The rate of change of the magnetic field (dB/dt) is indicative of the intensity of geomagnetically induced currents (GICs) flowing along electric power grid systems and other electrically conducting infrastructure, which can seriously affect normal operation.

Figure 12 shows an illustration included in the space weather bulletin tailored for the Venus Express aero braking operations.

\section{Future Perspectives of the Network}

The SWE network is foreseen to undergo extensive development within the next years. At first, the basis for the sustainability of each ESC will be set by compiling a dedicated definition and development plan based on the existing SWE customer requirements, system requirements and service development roadmaps. This will ensure the consistent presentation of structured SWE services to the end users built on the wide range of European expertise and assets available, coupled with targeted developments. Constant user feedback and a programme of test campaigns will be used to highlight areas where user requirements are evolving. Second, substantial development of existing and new products will enlarge the capabilities of each ESC. Further product development activities are expected to be executed within other ESA and EU programmes. As these developments mature, inclusion of relevant existing new or updated products into each ESC will be investigated in terms of their possible further contribution to meeting SWE user requirements. Following this assessment, their inclusion into the SWE network may be foreseen. 
Citation: Donder ED, Crosby N, Kruglanski M, Andries J, Devos A, et al. (2017) Services for Space Mission Support Within The ESA Space Situational Awareness Space Weather Service Network. J Aeronaut Aerospace Eng 6: 180. doi: 10.4172/2168-9792.1000180

Page 10 of 11

\begin{tabular}{|c|c|c|}
\hline Product & Description & Provider \\
\hline Magnetogrammes from North (west) Europe and Greenland & Real-time ground based vector measurements (Include Greenland and Finland) & $\begin{array}{l}\text { TGO/DTU } \\
\text { FMI }\end{array}$ \\
\hline Provisional K-indices from Northwest Europe & -- & TGO/DTU \\
\hline $\begin{array}{l}\text { Geomagnetic Activity index for } \\
\text { Aurora zone (AZ), last } 33 \text { days }\end{array}$ & -- & TGO \\
\hline $\begin{array}{l}\text { Geomagnetic Activity index for auroral zone (AZ), long term } \\
\text { variation }\end{array}$ & -- & TGO \\
\hline Provisional AA index & -- & SIDC/ROB \\
\hline Aurora alert and forecast service & Forecast of visible aurorae & FMI \\
\hline Quick look Kp index & Near Real-time Kp index plot & GFZ \\
\hline Most recent definitive Kp index & Kp musical diagram & GFZ \\
\hline Kp and Ap index on tabular form & $\begin{array}{l}\text { Latest quicklook } \mathrm{Kp} \text { and } \mathrm{Ap} \text { on tabular } \\
\text { form }\end{array}$ & GFZ \\
\hline Kp and $A p$ index archive & Historic Kp and Ap ftp database & GFZ \\
\hline Maps for power and pipeline operators & $\begin{array}{l}\text { Maps showing estimated GIC, E- fields } \mathrm{dB} / \mathrm{dt} \text { and } \mathrm{B} \text { in European model grid as } \\
\text { well as user specified grid }\end{array}$ & FMI, PGI \\
\hline Table of modelled GIC & $\begin{array}{l}\text { Tailored service for specific users providing a table of modelled GIC value for the } \\
\text { users network in the last minute and peak GIC in the last } 60 \text { mins. }\end{array}$ & FMI \\
\hline Forecasts of $\mathrm{dB} / \mathrm{dT}$ & $\sim 30$ min ahead & IRF \\
\hline PSP difference & $\begin{array}{l}\text { Tailored service for specific users providing pipe-to-soil potential difference (PSP) } \\
\text { variations in the users pipe network }\end{array}$ & FMI \\
\hline 27 day local forecast issued every 24 hours & $\begin{array}{l}\text { Forecast of local, } 24 \text { hour activity index using ARMA (CME override; ENLIL and/ } \\
\text { or Graz model may be used to improve the forecast in a later update) }\end{array}$ & TGO \\
\hline $\begin{array}{l}\text { Next } 24 \text { hours and next } 24-48 \text { hours local forecast issued } \\
\text { every hour }\end{array}$ & $\begin{array}{l}\text { Sliding forecast of local, } 24 \text { hour activity index using ARM (CME override; ENLIL } \\
\text { and/or Graz model may be used to improve the forecast in a later update) }\end{array}$ & TGO \\
\hline 27 day global forecast issued every 24 hours & $\begin{array}{l}\text { Forecast of Ap index using ARMA (CME override; ENLIL and/or Graz model may } \\
\text { be used to improve the forecast in a later update) }\end{array}$ & TGO \\
\hline Short term (1 hour) Kp forecast & Forecast using MAK method. & TGO \\
\hline Short term (1 hour) local geomagnetic forecast & Forecast using GAFS method & TGO \\
\hline $\begin{array}{l}\text { Real-time and historic geomagnetic activity plots and data } \\
\text { files for geomagnetic surveying (Total field) }\end{array}$ & $\begin{array}{l}\text { Based on data from } \\
\text { Norwegian } \\
\text { magnetometer } \\
\text { network }\end{array}$ & TGO \\
\hline $\begin{array}{l}\text { Real-time and historic geomagnetic activity plots and data } \\
\text { files for directional drilling (Total field, declination and } \\
\text { inclination) }\end{array}$ & Based on data from selected stations in Norwegian magnetometer network & TGO \\
\hline E-mail alerts for geomagnetic disturbances & $\begin{array}{l}\text { User receives e-mail when activity reaches certain threshold in a particular } \\
\text { region. }\end{array}$ & TGO \\
\hline
\end{tabular}

Table 1: List of G-ESC products.

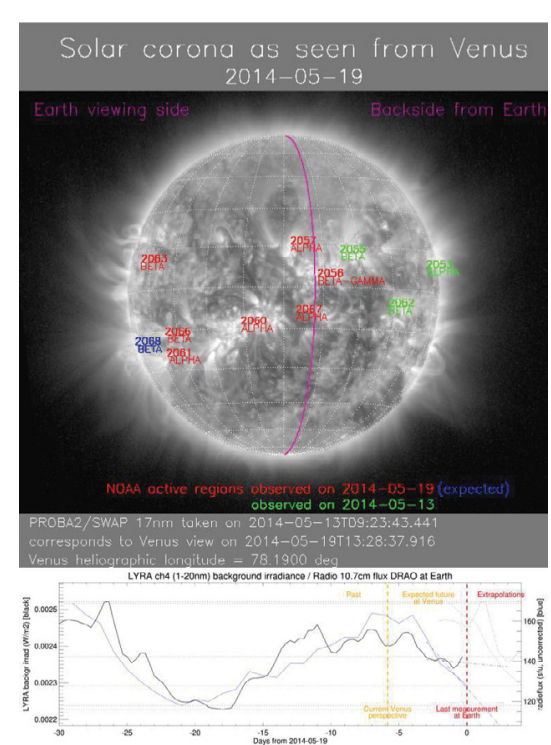

Figure 12: Illustration of a space weather bulletin for Venus Express operations showing an extrapolation of the solar disk image, EUV background irradiation and F10.7 index matching Venus viewpoint. 
Citation: Donder ED, Crosby N, Kruglanski M, Andries J, Devos A, et al. (2017) Services for Space Mission Support Within The ESA Space Situational Awareness Space Weather Service Network. J Aeronaut Aerospace Eng 6: 180. doi: 10.4172/2168-9792.1000180

\section{Conclusion}

The ESA SSA SWE programme is driving the development of a system to monitor, predict and disseminate space weather information and alerts, including products and services available for use by both space an ground based user communities. This development process is guided by regular assessments of the requirements and of the product quality, with end users in the loop. The challenge of this process is to understand the user needs and to translate them into targeted services providing accurate and timely space weather information. The space community is therefore invited to contribute with feedback and recommendations.

\section{Acknowledgment}

This paper has been prepared in the frame of the ESA Contract No. 4000112444/14/D/MRP.

\section{References}

1. Colorado B (2015) ESA SSA space weather service system. Juha-Pekka Luntama Space Weather Workshop.

2. SSA-SWE (2011) Space weather customer requirements document. SSASWE-RS-CRD-1001.

3. SSA-SWE (2013) Space weather system requirements document. SSA-SWERS-RD-0001.

4. Robbrecht E, Berghmans D (2004) Automated recognition of coronal mass ejections (CMEs) in near-real-time data, Astronomy and Astrophysics 425: 1097-1106.

5. Robbrecht E, Berghmans D, Van Der Linden RAM (2009) Automated LASCO CME Catalog for solar cycle 23: are CMEs scale invariant? The Astrophysical Journal 691: 1222-1234.

6. Georgoulis P, Manolis K, Rust C, David M (2007) Quantitative forecasting of major solar flares, The Astrophysical Journal 661: L109-L112.

7. Georgoulis MK (2011) Pre-eruption magnetic configurations in the activeregion solar photosphere. The Physics of Sun and Star Spots Proceedings of the International Astronomical Union IAU Symposium 273: 495-498.
8. Georgoulis MK (2010) Towards an efficient prediction of solar flares: Which parameters and how? Entropy 15: 5022-5052.

9. Odstrcil D, Pizzo VJ, Arge CN (2005) Propagation of the 12 May 1997 interplanetary coronal mass ejection in evolving solar wind structures Geophysical Research: Space Physics.

10. Vrsnak B, Temmer M, Veronig AM (2007) Coronal holes and solar wind highspeed streams: I Forecasting the solar wind parameters. Solar Physics 240: 315-330.

11. Zic T, Vrsnak B, Temmer M (2015) Heliospheric propagation of coronal mass ejections: Drag-based model fitting. The Astrophysical Journal Supplement Series 218: 7 .

12. Souvatzoglou G, Papaioannou A, Mavromichalaki H, Dimitroulakos J, Sarlanis C (2014) Optimizing the real-time ground level enhancement alert system based on neutron monitor measurements: Introducing GLE Alert Plus. Space Weather 12: 633-649.

13. Latocha M, Bek P, Rollet S (2009) AVIDOS a software package for European accredited aviation dosimetry Oxford Journals Science \& Mathematics Radiation Protection Dosimetry 136: 286-290.

14. Jakowski N, Mayer C, Borries C, Wilken V (2009) Space weather monitoring by ground and space based GNSS measurements Proc. ION - International Technical Meeting Anaheim CA.

15. SWE (2011) Service Validation Report (SWE-DELI5-0001).

16. Tsagouri I, Belehaki A (2008) An upgrade of the solar wind driven empirical model for the middle latitude ionospheric storm time response. J Atmos So Terr Phys.

17. Tsagouri I, Zolesi B, Belehaki A, Cander LR (2005) Evaluation of the performance of the real-time updated Simplified lonospheric Regional Model for the European area. J Atmos Sol-Terr Phys 67: 1137-1146.

18. Zolesi B, Belehaki A, Tsagouri I, Cander LR (2011) Real-time updating of the simplified ionospheric regional model for operational applications. Radio Science 39: 2.

19. Wintoft P, Wik M, Viljanen A (2015) Solar wind driven empirical forecast models of the time derivative of the ground magnetic field. J Space Weather Space Clim 5: 9.

20. Mc Pherron RL (1999) Predicting the Ap index from past behaviour and solar wind velocity. Phys Chem. Earth (C) 24: 45-56. 